bioRxiv preprint doi: https://doi. org/10.1101/2021.09.28.462109. this version posted September 29, 2021. The copyright holder for this preprint (which was not certified by peer review) is the author/funder, who has granted bioRxiv a license to display the preprint in perpetuity. It is made available under aCC-BY-NC-ND 4.0 International license.

\title{
1 Use of eVLP-based vaccine candidates to broaden immunity against SARS-CoV-2 variants
}

2 Jasminka Bozic ${ }^{\mathrm{a} 1}$, Tanvir Ahmed ${ }^{\mathrm{a} 1}$, Barthelemy Ontsouka ${ }^{\mathrm{a}}$, Anne-Catherine Fluckiger ${ }^{\mathrm{b}}$, Abebaw

3 Diress $^{a}$, Tamara Berthoud ${ }^{a}$, Xiaoyang Yuan, Lanjian Yang ${ }^{a}$, Francisco Diaz-Mitomac, David E.

4 Anderson $^{\mathrm{c}}$, Catalina Soare ${ }^{\mathrm{a} 1}$

5 aVBI Vaccines, 201-310 Hunt Club Road, Ottawa ON K1V 1C1, Canada; 'bionaria, 11Bis Rue

6 de la Garenne, 69290 Saint-Genis-les-Ollières, France; 'VBI Vaccines, Cambridge, 222

7 Third Street, Cambridge, 02142 Massachusetts, USA.

$8{ }^{*}$ Corresponding author:

9 Email: afluckiger@vbivaccines.com (A.-C. Fluckiger)

10 1: These authors contributed equally to this work.

\section{Abstract}

12 Rapid emergence of SARS-CoV-2 variants is a constant threat and a major hurdle to reach heard

13 immunity. We produced VBI-2905a, an enveloped virus-like particle (eVLP)-based vaccine

14 candidate expressing prefusion spike protein from the Beta variant that contains several escape

15 mutations. VBI-2905a protected hamsters against infection with a Beta variant virus and induced

16 high levels of neutralizing antibodies against Beta RBD. In a heterologous vaccination regimen, a

17 single injection of VBI-2905a in animals previously immunized with VBI-2902, a vaccine candidate

18 expressing S from ancestral SARS-CoV-2, hamsters were equally protected against Beta variant

19 infection. As an alternate strategy to broaden immunity, we produced a trivalent vaccine expressing

20 the prefusion spike protein from SARS-CoV-2 together with unmodifed S from SARS-CoV-1 and

21 MERS-CoV. Relative to immunity induced against the ancestral strain, the trivalent vaccine VBI-

22 2901a induced higher and more consistent antibody binding and neutralizing responses against a

23 panel of variants including Beta, Delta, Kappa, and Lambda, with evidence for broadening of

24 immunity rather than just boosting cross-reactive antibodies. 


\section{Keywords}

26 SARS-COV-2 variants; Vaccine; Virus-like-particles; Immunogenicity; cross-neutralizing antibodies

\section{Abbreviations}

28 eVLP, enveloped virus-like particules; CoV, coronavirus; VOC, Variant of concern; VOI, varaint of

29 interest; RBD, receptor binding domain; NTD, N-terminal domain; Ab, antibody; nAb, neutralizing 30 antibody; MLV, murine leukemia virus; ELISA, enzyme-linked-immuno-sorbent-assay; PRNT, 31 plaque reduction neutralization test; EPT, end-point titer; Alum, aluminum phosphate; IP, 32 IntraPeritoneal; IM, IntraMuscular; NRC, National Research Council Canada; VIDO,

33 Vaccine and Infectious Disease Organization

\section{Introduction}

The outbreak of a severe respiratory disease in Wuhan, China in December 2019 led to the identification of a new betacoronavirus related to the severe acute respiratory syndrome (SARS) coronavirus that was named SARS-CoV-2 (Wu et al., 2020). SARS-CoV-2 rapidly spread worldwide in a global pandemic in 2019 (COVID-19) and was declared a public health emergency of international concern (World Health Organization, 2020). Unprecedented effort and innovation in vaccine development resulted in vaccines, deployed under emergency use authorization, against SARS-CoV-2 in less than a year (FDA, 2020).

Coronaviruses are large single-strand RNA viruses with replication that is error-prone despite some proofreading mechanisms (Smith and Denison, 2012). Resulting mutational changes can either be detrimental and lead to viral extinction or confer advantage to the virus and result in better adaptation to the host. Massive replication of SARS-CoV-2 on a global scale contributes to increasing numbers of mutations and emergence of variants. Variants of concern (VOC) are defined by clear evidence indicating a significant impact on transmissibility, severity, and/or immunity that is likely to have an impact on the disease epidemiology (EDCC, 2021). In July 2021, four VOC that first emerged as locally dominant variants before spreading globally were discovered 
bioRxiv preprint doi: https://doi.org/10.1101/2021.09.28.462109; this version posted September 29, 2021. The copyright holder for this preprint (which was not certified by peer review) is the author/funder, who has granted bioRxiv a license to display the preprint in perpetuity. It is made available under aCC-BY-NC-ND 4.0 International license.

51 recently in India (B.1.617 - "Delta"). In late August the Mu variant first emerged in Columbia,

52 joining this list of Variants of Interest (VOI) together with Lambda while the incidence of Alpha was

53 decreasing.

The CoV spike (S) protein contains a receptor binding domain (RBD) critical for binding to

55 and infection of host cells, and is a major target for mutational changes which enhance adaptation

56 to the host (Berrio et al., 2020; Boni et al., 2020). Each of the VOC are characterized by a number

57 of shared mutations expressed on S, primarily located in the RBD and N-terminal domain (NTD),

58 that serve to increase inter-individual transmission, escape neutralizing antibodies acquired by

59 vaccination or prior natural SARS-CoV-2 infection, or both (Harvey et al., 2021; Zhang et al.,

60 2020). For instance, the first identified VOC, Alpha, is characterized by a D614G mutation, among

61 other mutations, that is now fixed in all globally circulating variants of the virus. D614G is

62 associated with increased transmissibility (Plante et al., 2021; Zhang et al., 2020) but does not

63 have a major impact on neutralization by serum from either vaccinated or COVID-19 convalescent

64 individuals. The following VOC that emerged in South Africa was rapidly identified as a vaccineescape mutant. This Beta variant bears several mutations in its RBD, including E484K and N501Y, which significantly inhibit neutralizing activity elicited against the Ancestral Wu-1 strain of virus whether acquired by vaccination or infection (Tegally et al., 2020; Cele et al., 2021). Emergence of escape mutants is a major concern because most of the licensed vaccines are based on expression of various forms of $S$ using the Ancestral sequence of the $S$ protein (Hoffmann et al., 2021 ; Kyriakidis et al., 2021; Lamb, 2021). More recently, the Delta variant spread from India to many countries with great speed in spite of significant proportions of fully vaccinated invididuals in many countries. Delta shows the RBD mutation L452R which appeared independently in several areas of the globe, including in variants Lambda, Kappa, Epsilon, lota, and contributes to escape neutralization from Abs induced by previously acquired immunity (Deng et al.2021). Additionally, mutation P681R in the furin cleavage site of Delta could increase the rate of S1-S2 cleavage, resulting in better transmissibility (Cherian et al., 2021).

Recently, we developed a SARS-CoV-2 candidate vaccine, VBI-2902a, comprised of enveloped virus-like particles (eVLPs) expressing a modified prefusion form of the ancestral $\mathrm{S}$ sequence, adjuvanted with aluminum phosphate (Alum). We recently demonstrated that VBI-2902a 
80 induced strong neutralizing activity in mouse immunogenicity studies, and protected hamsters from

81 SARS-CoV-2 challenge using a virus related to the ancestral isolate (Fluckiger et al. 2021). Interim

82 results from a Phase I clinical study in healthy, seronegative individuals (ClinicalTrials.gov

83 Identifier: NCT04773665) demonstrated robust (4.3-fold greater) neutralizing activity 28 days after

84 a second, $5 \mu \mathrm{g}$ dose of VBI-2902a, relative to a panel of COVID-19 convalescent sera.

Employing the same strategy, we produced a new vaccine candidate, VBI-2905a, that expresses a modified prefusion $S$ based on the Beta variant sequence. Consistent with previous studies, VBI-2905a elicited neutralizing antibody responses against the Beta variant which were significantly greater than those induced by VBI-2902a, and responses against the ancestral strain which were comparable to VBI-2902a. Consistent with the role of neutralizing antibody responses

90 as a presumed correlate of protection, greater efficacy was observed in hamsters vaccinated with

$91 \mathrm{VBI}-2905 a$ relative to $\mathrm{VBI}-2902 a$ when challenged with the Beta variant. Noteworthy was the

92 observation in an alternative vaccination regimen that priming with VBI-2902a followed by a single

93 booster dose of VBI-2905a induced strong neutralizing antibody responses against the ancestral

94 strain as well as both Beta and Delta VOC.

We also evaluated immunity elicited with a distinct eVLP-based candidate, VBI-2901a, which expresses a modified prefusion $S$ based on the ancestral sequence in addition to the related S proteins from SARS CoV-1 and MERS. Immunization with VBI-2901a induced neutralizing antibody titers against the Beta variant significantly greater than VBI-2902a and comparable to those induced with VBI-2905a, effectively broadening immunity to VOC not contained within the vaccine. Antibody binding and neutralizing titers against an extended panel of variants demonstrated responses typically 3-fold greater than that observed with VBI-2902a. Collectively, these results demonstrate multiple ways to broaden immunity to SARS-CoV-2 VOC.

\section{Material and Methods}


105 Expression plasmid for the production of eVLPs expressing SARS-CoV-2 S proteins have been 106 described previously (Fluckiger et al. 2021). Briefly, the prefusion modified form of $S$ was obtained 107 by introducing a mutation at the furin cleavage site (RRAR $\rightarrow$ GSAS) and two Proline at position 108 K986-V987 of the Wuhan reference and swapping the transmembrane cytoplasmic domain with 109 that of the VSV-G protein. VBI-2902a was produced using the Wuhan-Hu-1 spike sequence 110 (Genbank accession number MN908947), and VBI-2905a was produced using the same strategy 111 with S sequence from Beta variant B.1.351 isolate EPI_ISL_911433 (GISAID). Production and

112 purification of eVLPs were conducted as described elsewhere (Fluckiger et al. 2021). The 113 preparation of eVLPs expressing either Wuhan reference Spike or Beta variant Spike were 114 formulated in Aluminum phosphate (Alum, Adjuphos ${ }^{\circledR}$, Invitrogen) to obtain vaccine candidate 115 VBI-2902a and VBI-2905a, respectively. To produce VBI-2901, Two additionnal plasmids were 116 produced that expressed the optimized sequences for full-lenght unmodified S protein from SARS117 CoV-1 and MERS-CoV. To produce trivalent eVLPs, HEK-293SF-3F6 were cotransfected with 118 these 2 plasmids together with the plasmid coding for prefusion ancestral SARS-CoV-2 S used for 119 VBI-2902a production, and the MLVGAG plasmid as described. Expression of SARS-CoV-2 S, 120 SARS-CoV-1 S, MERS-CoV S and GAG were determined by Western blot analysis 121 (Supplementary material Fig.S1).

\section{Mouse immunization study}

123 Six- to 8-week-old female C57BL/6 mice were purchased from Charles River (St Constant, 124 Quebec Canada). The animals were acclimatized for a period of at least 7 days before any 125 procedures were performed. The animal studies were conducted under ethics protocols approved 126 by the NRC Animal Care Committee. Mice were maintained in a controlled environment in 127 accordance with the "Guide for the Care and Use of Laboratory Animals" at the Animal Research 128 facility of the NRC's Human Health Therapeutics Research Centre (Montreal). Mice were randomly 129 assigned to experimental groups of 10 to 15 mice and received intraperitoneal (IP) injections with $1300.5 \mathrm{~mL}$ of adjuvanted SARS-CoV-2 eVLPs as described elsewhere (Fluckiger, 2021). Blood was 
131 collected on day -1 before injection and day 14 after each injection for humoral immunity

132 assessment at time of euthanasia.

\section{Hamster challenge study}

135 Syrian golden hamsters (males, 5-6 weeks old) were purchased from Charles River Laboratories

136 (Saint-Constant, Quebec, Canada). The study was conducted under approval of the CCAC

137 committee at the Vaccine and Infectious Disease Organization (VIDO) International Vaccine Centre

138 (Saskatchewan, Canada). Animals were randomly assigned to each experimental group (A, B)

139 ( $n=10$ /group). Animals received 2 intramuscular (IM) injection of either $0.9 \%$-saline buffer (saline

140 control group) or VBI-2902a (VBI-2902a group), or VBI-2905a (VBI-2905a group), or a first dose of

141 VBI-2902a followed by a second injection of VBI-2905a (Heterologous boost group). Each dose of

$142 \mathrm{eVLP}$-based vaccine contained $1 \mu \mathrm{g}$ of Spike protein formulated with $125 \mu \mathrm{g}$ of Alum. Injection was

143 performed by intramuscular (IM) route at one side of the thighs in a $100 \mu \mathrm{L}$ volume. The schedule

144 for immunization, challenge and sample collection is depicted on Fig. 2a. All animals were

145 challenged intranasally via both nares with $50 \mu \mathrm{L} /$ nare containing $1 \times 10^{5}$ TCID50 of hCoV-19/South

146 Africa/KRISP-EC-K005321/2020 (Seq. available at GISAID: EPI_ISL_678470) strain per animal.

147 Body weights and body temperature were measured at immunization for 3 days and daily from the

148 challenge day. General health conditions were observed daily through the entire study period.

149 Blood samples were collected as indicated on Fig. 2a.

150 Antibody binding titers

151 Anti-SARS-CoV-2 specific IgG binding titers in sera were measured by standard ELISA procedure 152 described elsewhere (Kirchmeier et al., 2014), using recombinant SARS-CoV-2 S RBD proteins 153 (Sinobiological). For total lgG binding titers, detection was performed using a goat anti-mouse lgG154 Fc HRP (Bethyl), or Goat anti-Hamster IgG HRP (ThermoFisher), or goat anti-human IgG heavy 155 and light chain HRP-conjugated (Bethyl). HRP-conjugated Goat anti-mouse IgG1 and HRP156 conjugated goat anti-mouse IgG2b HRP (Bethyl) were used for the detection of isotype subtype. 
Determination of $A b$ binding titers to Spike RBDs was performed using SARS-COV-2 RDB recombinant protein for the specificty of choice as described in Suppl. Table 1. The detection was completed by adding 3,3',5,5'-tetramethylbenzidine (TMB) substrate solution, and the reaction stopped by adding liquid stop solution for TMB substrate. Absorbance was read at $450 \mathrm{~nm}$ in an

161 ELISA microwell plate reader. Data fitting and analysis were performed with SoftMaxPro 5, using a 162 four-parameter fitting algorithm.

Virus neutralization assays

164 Neutralizing activity in mouse serum samples was measured by standard plaque reduction 165 neutralization test (PRNT) on Vero cells at the NRC using 100 PFU of 166 SARS-CoV-2/Canada/ON/VIDO-01/2020 (Wu-1 virus) or hCoV-19/South Africa/KRISP-EC167 K005321/2020 (Beta virus). Results were represented as PRNT90 end point titer (EPT), 168 corresponding to the lowest dilution inhibiting respectively $90 \%$ of plaque formation in Vero cell 169 culture.

Neutralization assay with pseudoparticles

172 Production of pseudoparticles ( $\mathrm{pp}$ ) pseudotyped with various spike proteins and neutralization 173 assay was adapted from Dreux et al, 2009. Expression plasmid were designed using full length $S$ 174 protein sequences as described previsouly. Accession number and mutations are listed in Suppl. 175 Material Table 1. We produced infectious SARS-CoV-2pp carrying a GFP-firefly luciferase double 176 reporter gene (plasmid pjm155, Garrone et al., 2011) instead of green fluorescent protein (GFP). 177 Luciferase activity in infected hACE2-HEK293 cells was measured with a Bright-Glo Luciferase 178 assay system (Promega) and a Beckman Coulter DTX880 plate reader. Data were expressed in 179 relative luminescence units (RLUs). The percentage of neutralization was calculated by comparing 180 the luciferase activity in cells infected with SARS-CoV-2pp in the presence of serum from 181 immunized animals with luciferase activity in cells infected with SARS-CoV-2pp in the absence of 182 serum. 


\section{Statistics}

184 All statistical analyses were performed using GraphPad Prism 9 software (La Jolla, CA). Unless indicated, multiple comparison was done with Dunn's corrected Kruskall-Wallis test on unpaired samples and Friedman test on paired samples. The data were considered significant if $p<0.05$. Geometric mean titers (GMT) with standard deviation are represented on graphs. No samples or animals were excluded from the analysis. Randomization was performed for the animal studies.

\section{Results}

Heterologous boosting with eVLPs bearing $S$ protein from the Beta variant broadens

\section{immunity}

193 VBI-2902a is an eVLP-based vaccine candidate that expresses a modified prefusion SARS-CoV-2

194 S protein from the ancestral Wu-1 strain, adjuvanted with Alum (Fluckiger et al., 2021). VBI-2905a expresses a modified prefusion SARS-CoV-2 $S$ protein from the Beta variant and is also adjuvanted with Alum. We immunized mice with 2 injections of VBI-2902a, 2 injections of VBI2905a, or a first injection of VBI-2902a followed by a second injection of VBI-2905a (heterologous boost). As previously described (Fluckiger et al., 2021), 2 doses of VBI-2902a induced high levels

199 of neutralizing $\mathrm{Ab}$ response against the ancestral $\mathrm{Wu}-1$ strain $(\mathrm{GMT}=2,458)$ which were 200 significantly reduced against the Beta variant $(\mathrm{GMT}=94)$ (Fig.1a-b). By contrast, VBI-2905a 201 induced Abs that neutralized Beta and ancestral viruses at similar levels in mice, yielding only a 202 2.2-fold difference with non significant $p=0.1484$ (Fig. 1a-b). Sera from mice in the heterologous 203 boost group cross-neutralized both the Beta variant and the ancestral strain with similar potencies $204(1,4$ fold difference with $p=0.3828)$. Heterologous boosting with VBI-2905a significantly increased 205 the PRNT90 against the ancestral strain compared to 2 doses of VBI-2905a alone (from GMT of 371 to $820, p=0.0267$ ) to levels that were closer to those reached after two doses of VBI-2902a 
bioRxiv preprint doi: https://doi.org/10.1101/2021.09.28.462109; this version posted September 29, 2021. The copyright holder for this preprint (which was not certified by peer review) is the author/funder, who has granted bioRxiv a license to display the preprint in perpetuity. It is made available under aCC-BY-NC-ND 4.0 International license.

207 ( $p=0.0131)$, while PRNT90 GMTs against the Beta variant were comparable to 2 doses of VBI-

$2082905 a$ (respectively GMT $=564$ vs $\mathrm{GMT}=619, p=0.8785$ ).

Analysis of $\mathrm{Ab}$ binding titers to $\mathrm{S}$ protein RBDs was consistent with the neutralization data

210 (Fig.1c). VBI-2902a induced high levels (most of the sera $>10^{6}$ EPT with GMT $974 \times 10^{3}$ ) of $A b$

211 binding titers against the ancestral S RBD with significantly reduced cross-reactivity against the

212 Beta variant RBD (GMT $\left.74 \times 10^{3}\right)$, though there was good cross-reactivity against the Delta variant

213 RBD (GMT 616 $\times 10^{3}$ ). Antisera from immunization with VBI-2905a showed similar crossreactivty

214 against Ancestral, Delta and Beta RBD (respectively GMT $322 \times 10^{3}, 192 \times 10^{3}$ and $217 \times 10^{3}$ ). Animals

215 receiving the heterologous prime boost regimen had similar reactivity to ancestral and Delta RBD

216 as compared with the VBI-2902a group, and similar reactivity to Beta RBD compared to the VBI-

$2172905 a$ group.

Heterologous boosting with VBI-2905a protects hamsters against SARS-COV-2 Beta variant

Golden Syrian hamsters were intramuscularly vaccinated 3 weeks apart with two doses of

eVLP vaccine candidates, comprised of: two doses of VBI-2902a (group VBI-2902a), two doses of

VBI-2905a (group VBI-2905a), or a priming dose of VBI-2902a followed by a second, booster dose of VBI-2905a (group heterologous boost) (Fig. 2a).

Neutralizing activities titers against the ancestral virus were comparable across all groups,

224 including hamsters immunized with 2 doses of the Beta $S$ candidate (VBI-2905a) (Fig.2b).

225 Neutralization of the Beta variant was lower after immunization with VBI-2902a, with a significant

226 9.6-fold decrease of Beta nAb compared to homotypic immunization with VBI-2905a (GMT 99 in $227 \mathrm{VBI}-2902 \mathrm{a}$ and 1083 in VBI-2905a, $\mathrm{p}=0$,0033). In contrast, nAb titers against Beta RBD were 228 similar in groups that received either two doses of VBI-2905a or heterologous boosting.

Three weeks after the second immunization, hamsters were exposed to $1 \times 10^{5}$ TCID50 of

230 the Beta variant virus in each nare. In the placebo group, hamsters began losing weight the day

231 after infection which continued until day 6-8. Vaccination with 2 doses of VBI-2902a based on the

232 ancestral $S$ protein induced limited protection against challenge with moderate weight loss

233 recorded until day 4 , and only a fraction (3/5) of the animals fully regained their initial body weight

234 after day 7. By contrast, hamsters vaccinated with 2 doses of VBI-2905a exhibited transient weight 
bioRxiv preprint doi: https://doi.org/10.1101/2021.09.28.462109. this version posted September 29, 2021. The copyright holder for this preprint (which was not certified by peer review) is the author/funder, who has granted bioRxiv a license to display the preprint in perpetuity. It is made available under aCC-BY-NC-ND 4.0 International license.

235 loss up to day 2-3 and then rapidly regained weight. A similar pattern was observed in hamsters

236 that received $\mathrm{VBI}-2905 \mathrm{a}$ as a boost. As we have observed in previous hamster challenge studies of

$237 \mathrm{VBI}-2902 \mathrm{a}$, there was a correlation between neutralizing antibody titers against the Beta variant 238 and protection from disease (weight loss) after challenge (data not shown).

Immunization with a pan-coronavirus candidate may protect against VOC not contained

\section{within the vaccine}

We hypothesized that exposing the immune system to multiple spike proteins at the same

242 time might help broaden humoral immunity that could recognize emerging variants or new

243 coronaviruses more phylogenetically distant to the vaccine candidate. To test this hypothesis we 244 produced VBI-2901a, a trivalent eVLP vaccine formulated with Alum, that expresses a prefusion 245 form of the ancestral SARS-CoV-2 S (identical to VBI-2902) with unmodified full length S from 246 SARS-CoV-1 and MERS-CoV (Suppl. Fig. S1). Mice that received 2 doses of trivalent VBI-2901a 247 had increased nAb titers (GMT 2915) against the ancestral virus relative to mice that received 2 248 doses of monovalent VBI-2902a (GMT 831) or VBI-2905a (GMT 448) (Fig. 3a). Moreover, trivalent 249 VBI-2901a induced neutralization activity against the Beta virus that was equivalent to what was observed in response to homotypic VBI-2905a, and significantly higher than that observed after VBI-2902a vaccination (Fig. 3a). Neutralization of both Delta and Kappa variant pseudotyped particles confirmed broadened neutralizing immunity elicited by VBI-2901a, with titers approximately 3-fold greater than those induced by VBI-2902a (Fig. 3b). Consistent with the neutralization activity, VBI-2901a induced higher and/or more consistent levels of Ab binding to the RBD among all variants evaluated, including Beta, Delta, and Lambda (Fig. 3c).

\section{Discussion}

Less than a year after identification of the new SARS-CoV-2 virus, variants emerged with impact on transmissibility, severity and immunity (EDCC, 2021) that challenge the development and durability of vaccine strategies designed to reach herd immunity. Indeed, all approved 
bioRxiv preprint doi: https://doi.org/10.1101/2021.09.28.462109; this version posted September 29, 2021. The copyright holder for this preprint (which was not certified by peer review) is the author/funder, who has granted bioRxiv a license to display the preprint in perpetuity. It is made available under aCC-BY-NC-ND 4.0 International license.

261 but has been replaced by variants containing mutations which are enabling escape from nAbs

262 induced against the ancestral strain (Berio et al. 2020; Boni et al, 2020). In the present study, we compared several strategies to broaden antibody-based immunity which is presumed to be a correlate of protection against SARS-CoV-2.

In addition to our eVLP vaccine expressing the prefusion S from the ancestral SARS-CoV-2 virus, we produced an eVLP-based vaccine expressing the prefusion S from Beta variant. Beta was chosen for its deleterious mutations E484K and K417N, which enable escape neutralization from ancestral virus mAbs (Hoffman et al, 2021). We have previously demonstrated that 2 doses of VBI-2902a protected hamsters against infection by the ancestral Wuhan SARS-CoV-2 virus and we confirmed here that VBI-2905a also protected hamsters from infection with the SARS-CoV-2 Beta variant. We have also demonstrated that a heterologous boost with Beta variant vaccine VBI2905a given to animals that had received a single priming dose of ancestral strain vaccine VBI2902a protected against the new Beta variant while also maintaining cross-reactivity against the ancestral strain Moreover, heterologous eVLP boosting with VBI-2905a also induced high levels of antibody reactivity against the globally dominant Delta VOC. Additional challenge studies are in progress to evaluate if a heterologous boosting strategy can confer protection in Syrian golden hamsters against infection with the Delta variant.

Building upon the flexibility of the eVLP vaccine technology, we produced VBI-2901a, a multivalent coronavirus candidate containing $S$ proteins from SARS-CoV-2, SARS-CoV, and MERS-CoV with the intent to broaden immunity to emerging VOC as well as novel, related betacoronaviruses that may infect humans in the future. Vaccines currently in use or in clinical evaluation that are based on the ancestral strain induce neutralizing antibody responses that are less reactive against the Beta VOC, with titers typically 5-10 lower than against the ancestral strain 284 (Wibmer et al., 2021; Wang et al., 2021). In marked contrast, VBI-2901a elicited robust nAb responses not only against the ancestral SARS-CoV-2 strain, but also against the Beta variant, providing evidence of the vaccine candidate's ability to broaden immunity and "anticipate" an emerging variant not contained within the vaccine. High levels of cross-neutralizing activity elicited by VBI-2901a were also observed against the Delta and Kappa variants. Other studies have shown 
bioRxiv preprint doi: https://doi.org/10.1101/2021.09.28.462109; this version posted September 29, 2021. The copyright holder for this preprint (which was not certified by peer review) is the author/funder, who has granted bioRxiv a license to display the preprint in perpetuity. It is made available under aCC-BY-NC-ND 4.0 International license.

290

291

292

293

294

295

296

mRNA vaccine, which is based on the ancestral SARS-CoV-2 virus, contained a broad spectrum of neutralizing antibodies against 10 sarbecoviruses tested, including SARS-COV-2 variants, several strains of SARS-CoV-1, and Bat and Pangolin CoV (Tan et al., 2021). Further studies are underway to better understand how VBI-2901a, which similarly exposes the B cell repertoire to spike proteins from both SARS-CoV-1 and SARS-CoV-2, broadens neutralizing activity against SARS-CoV-2 variants as well as to assess neutralizing responses to phylogenetically more distant coronaviruses.

Broadening of the neutralizing antibody response has also been shown using nanoparticles of mosaïc RBD from various betacoronavirus species (Cohen et al., 2021; Walls et al., 2021). However, the $\mathrm{N}$ terminal domain of the $\mathrm{S}$ protein is another important target for neutralizing antibodies and the site of many mutations that could potentially contribute to antibody neutralization escape (Andreoni et al. 2021). Given that VBI-2901a expresses the full-length ectodomain of the Coronaviruses spike, it will be critical to determine the respective roles and importance of the RBD, NTD, and the highly conserved S2 domains in broadening immunity.

Whereas vaccines based on the ancestral strain of SARS-CoV-2 protect against severe disease caused by variants of concern, variants such as Beta are less sensitive to vaccine-induced immunity and efficacy rates are accordingly lower. This is likely to become more apparent as vaccine-induced immunity wanes and as variants continue to emerge with even greater numbers of mutations. One strategy to address these concerns is to administer booster doses to increase neutralizing antibody titers against the ancestral strain, a subset of which may cross-neutralize variants of concern. We have described three alternate strategies that have the potential to broaden immunity to a greater extent. An eVLP-based candidate based on the Beta variant $S$ protein, VBI-2905a, induces potent immunity against not just the Beta virus, but also against the ancestral strain, though it is less potent against the Delta variant. However, building upon immunity induced against the ancestral strain with a priming dose of $\mathrm{VBI}-2902 \mathrm{a}$, a single booster dose of VBI-2905a resulted in potent and more balanced neutralizing antibody responses against the ancestral virus, and Beta and Delta variants. Finally, we have described a novel trivalent eVLP candidate, VBI-2901a, which elicited potent and broad immunity against all variants tested, 
318 including Beta, Delta, Lambda, and Kappa, with the testing for the potential to neutralize more

319 distantly related viruses currently underway.

\section{Acknowledgment}

321 The authors want to thank Adam Asselin, Matthew Yorke, Teresa Daoud, Rebecca Wang, Gillian

322 Lampkin (VBI vaccines) for outstanding technical support; NRC Animal Resources Group and the

323 VIDO Saskatchewan team for remarkable care with animal experiments.

\section{Funding}

325 The work performed in this manuscript was supported by the Coalition for Epidemic Preparedness

326 Innovations (CEPI)

\section{References}

2. Berrio A, Gartner V, Wray GA. Positive selection within the genomes of SARS-CoV-2 and other Coronaviruses independent of impact on protein function. PeerJ. 2020 Oct 16;8:e10234. doi: 10.7717/peerj.10234. PMID: 33088633; PMCID: PMC7571416.

1. Andreano E, Piccini G, Licastro D, Casalino L, Johnson NV, Paciello I, Dal Monego S, Pantano E, Manganaro N, Manenti A, Manna R, Casa E, Hyseni I, Benincasa L, Montomoli E, Amaro RE, McLellan JS, Rappuoli R. SARS-CoV-2 escape from a highly neutralizing COVID-19 convalescent plasma. Proc Natl Acad Sci U S A. 2021 Sep 7;118(36):e2103154118. doi: 10.1073/pnas.2103154118. PMID: 34417349.

3. Boni MF, Lemey P, Jiang X, Lam TT, Perry BW, Castoe TA, Rambaut A, Robertson DL. Evolutionary origins of the SARS-CoV-2 sarbecovirus lineage responsible for the COVID-19 pandemic. Nat Microbiol. 2020 Nov;5(11):1408-1417. doi: 10.1038/s41564-020-0771-4. Epub 2020 Jul 28. PMID: 32724171.

4. Cele S, Gazy I, Jackson L, Hwa SH, Tegally H, Lustig G, Giandhari J, Pillay S, Wilkinson E, Naidoo Y, Karim F, Ganga Y, Khan K, Bernstein M, Balazs AB, Gosnell BI, Hanekom W, Moosa MS; Network for Genomic Surveillance in South Africa; COMMIT-KZN Team, 
Lessells RJ, de Oliveira T, Sigal A. Escape of SARS-CoV-2 501Y.V2 from neutralization by convalescent plasma. Nature. 2021 May;593(7857):142-146. doi: 10.1038/s41586-02103471-w. Epub 2021 Mar 29. PMID: 33780970.

5. Cherian S, Potdar V, Jadhav S, Yadav P, Gupta N, Das M, Rakshit P, Singh S, Abraham P, Panda S, Team N. SARS-CoV-2 Spike Mutations, L452R, T478K, E484Q and P681R, in the Second Wave of COVID-19 in Maharashtra, India. Microorganisms. 2021 Jul 20;9(7):1542. doi: 10.3390/microorganisms9071542. PMID: 34361977; PMCID: PMC8307577.

6. Cohen AA, Gnanapragasam PNP, Lee YE, Hoffman PR, Ou S, Kakutani LM, Keeffe JR, Wu HJ, Howarth M, West AP, Barnes CO, Nussenzweig MC, Bjorkman PJ. Mosaic nanoparticles elicit cross-reactive immune responses to zoonotic coronaviruses in mice. Science. 2021 Feb 12;371(6530):735-741. doi: 10.1126/science.abf6840. Epub 2021 Jan 12. PMID: 33436524; PMCID: PMC7928838.

7. Deng X, Garcia-Knight MA, Khalid MM, Servellita V, Wang C, Morris MK, SotomayorGonzález A, Glasner DR, Reyes KR, Gliwa AS, Reddy NP, Sanchez San Martin C, Federman S, Cheng J, Balcerek J, Taylor J, Streithorst JA, Miller S, Sreekumar B, Chen PY, Schulze-Gahmen U, Taha TY, Hayashi JM, Simoneau CR, Kumar GR, McMahon S, Lidsky PV, Xiao Y, Hemarajata P, Green NM, Espinosa A, Kath C, Haw M, Bell J, Hacker JK, Hanson C, Wadford DA, Anaya C, Ferguson D, Frankino PA, Shivram H, Lareau LF, Wyman SK, Ott M, Andino R, Chiu CY. Transmission, infectivity, and neutralization of a spike L452R SARS-CoV-2 variant. Cell. 2021 Jun 24;184(13):3426-3437.e8. doi: 10.1016/j.cell.2021.04.025. Epub 2021 Apr 20. PMID: 33991487; PMCID: PMC8057738.

8. Dreux M, Cosset FL. Detection of neutralizing antibodies with HCV pseudoparticles (HCVpp). Methods Mol Biol. 2009;510:427-38. doi: 10.1007/978-1-59745-394-3_32. PMID: 19009280.

9. Fluckiger AC, Ontsouka B, Bozic J, Diress A, Ahmed T, Berthoud T, Tran A, Duque D, Liao M, McCluskie M, Diaz-Mitoma F, Anderson DE, Soare C. An enveloped virus-like particle vaccine expressing a stabilized prefusion form of the SARS-CoV-2 spike protein elicits 
10.1016/j.vaccine.2021.07.034. Epub 2021 Jul 16. PMID: 34304928; PMCID: PMC8282453.

10. Garrone P, Fluckiger AC, Mangeot PE, Gauthier E, Dupeyrot-Lacas P, Mancip J, Cangialosi

Klatzmann D, Dalba C. A prime-boost strategy using virus-like particles pseudotyped for HCV proteins triggers broadly neutralizing antibodies in macaques. Sci Transl Med. 2011 Aug 3;3(94):94ra71. doi: 10.1126/scitranslmed.3002330. PMID: 21813755.

11. Harvey WT, Carabelli AM, Jackson B, Gupta RK, Thomson EC, Harrison EM, Ludden C,

Reeve R, Rambaut A; COVID-19 Genomics UK (COG-UK) Consortium, Peacock SJ,

Robertson DL. SARS-CoV-2 variants, spike mutations and immune escape. Nat Rev Microbiol. 2021 Jul;19(7):409-424. doi: 10.1038/s41579-021-00573-0. Epub 2021 Jun 1. PMID: 34075212; PMCID: PMC8167834.

12. Hoffmann M, Arora P, Groß R, Seidel A, Hörnich BF, Hahn AS, Krüger N, Graichen L, Hofmann-Winkler H, Kempf A, Winkler MS, Schulz S, Jäck HM, Jahrsdörfer B,

B.1.351 and P.1 escape from neutralizing antibodies. Cell. 2021 Apr 29;184(9):23842393.e12. doi: 10.1016/j.cell.2021.03.036. Epub 2021 Mar 20. PMID: 33794143; PMCID: PMC7980144.

13. European Centre for Disease Prevention and Control, SARS-Co-V-2 variants of concern as of 23 september 2021; https://www.ecdc.europa.eu/en/covid-19/variants-concern

15. World Health Organization, Statement on the second meeting of the International Health Regulations (2005) Emergency Committee regarding the outbreak of novel coronavirus 
16. Kirchmeier M, Fluckiger AC, Soare C, Bozic J, Ontsouka B, Ahmed T, Diress A, Pereira L, Schödel F, Plotkin S, Dalba C, Klatzmann D, Anderson DE. Enveloped virus-like particle expression of human cytomegalovirus glycoprotein B antigen induces antibodies with potent and broad neutralizing activity. Clin Vaccine Immunol. 2014 Feb;21(2):174-80. doi: 10.1128/CVI.00662-13. Epub 2013 Dec 11. PMID: 24334684; PMCID: PMC3910943.

17. Kyriakidis NC, López-Cortés A, González EV, Grimaldos AB, Prado EO. SARS-CoV-2 vaccines strategies: a comprehensive review of phase 3 candidates. NPJ Vaccines. 2021 Feb 22;6(1):28. doi: 10.1038/s41541-021-00292-w. PMID: 33619260; PMCID: PMC7900244.

18. Lamb YN. BNT162b2 mRNA COVID-19 Vaccine: First Approval. Drugs. 2021 Mar;81(4):495-501. doi: 10.1007/s40265-021-01480-7. PMID: 33683637; PMCID: PMC7938284.

19. Plante JA, Liu Y, Liu J, Xia H, Johnson BA, Lokugamage KG, Zhang X, Muruato AE, Zou J, Fontes-Garfias CR, Mirchandani D, Scharton D, Bilello JP, Ku Z, An Z, Kalveram B, Freiberg AN, Menachery VD, Xie X, Plante KS, Weaver SC, Shi PY. Spike mutation D614G alters SARS-CoV-2 fitness. Nature. 2021 Apr;592(7852):116-121. doi: 10.1038/s41586-0202895-3. Epub 2020 Oct 26. Erratum in: Nature. 2021 Jul;595(7865):E1. PMID: 33106671; PMCID: PMC8158177.

20. Smith EC, Denison MR. Implications of altered replication fidelity on the evolution and pathogenesis of coronaviruses. Curr Opin Virol. 2012 Oct;2(5):519-24. doi: 10.1016/j.coviro.2012.07.005. Epub 2012 Aug 1. PMID: 22857992; PMCID: PMC7102773.

21. Tegally H, Wilkinson E, Giovanetti M, Iranzadeh A, Fonseca V, Giandhari J, Doolabh D, Pillay S, San EJ, Msomi N, Mlisana K, von Gottberg A, Walaza S, Allam M, Ismail A, Mohale T, Glass AJ, Engelbrecht S, Van Zyl G, Preiser W, Petruccione F, Sigal A, Hardie D, Marais G, Hsiao NY, Korsman S, Davies MA, Tyers L, Mudau I, York D, Maslo C, Goedhals D, Abrahams S, Laguda-Akingba O, Alisoltani-Dehkordi A, Godzik A, Wibmer CK, Sewell BT, Lourenço J, Alcantara LCJ, Kosakovsky Pond SL, Weaver S, Martin D, Lessells RJ, Bhiman JN, Williamson C, de Oliveira T. Detection of a SARS-CoV-2 variant of concern in 
South Africa. Nature. 2021 Apr;592(7854):438-443. doi: 10.1038/s41586-021-03402-9. Epub 2021 Mar 9. PMID: 33690265.

22. Wang R, Chen J, Gao K, Wei GW. Vaccine-escape and fast-growing mutations in the United Kingdom, the United States, Singapore, Spain, India, and other COVID-19devastated countries. Genomics. 2021 Jul;113(4):2158-2170. doi: 10.1016/j.ygeno.2021.05.006. Epub 2021 May 15. PMID: 34004284; PMCID: PMC8123493.

23. Wibmer CK, Ayres F, Hermanus T, Madzivhandila M, Kgagudi P, Oosthuysen B, Lambson BE, de Oliveira T, Vermeulen M, van der Berg K, Rossouw T, Boswell M, Ueckermann V, Meiring S, von Gottberg A, Cohen C, Morris L, Bhiman JN, Moore PL. SARS-CoV-2 501Y.V2 escapes neutralization by South African COVID-19 donor plasma. Nat Med. 2021 Apr;27(4):622-625. doi: 10.1038/s41591-021-01285-x. Epub 2021 Mar 2. PMID: 33654292. 24. Wu F, Zhao S, Yu B, Chen YM, Wang W, Song ZG, Hu Y, Tao ZW, Tian JH, Pei YY, Yuan ML, Zhang YL, Dai FH, Liu Y, Wang QM, Zheng JJ, Xu L, Holmes EC, Zhang YZ. A new coronavirus associated with human respiratory disease in China. Nature. 2020 Mar;579(7798):265-269. doi: 10.1038/s41586-020-2008-3. Epub 2020 Feb 3. Erratum in: Nature. 2020 Apr;580(7803):E7. PMID: 32015508; PMCID: PMC7094943.

25. Zhang L, Jackson CB, Mou H, Ojha A, Peng H, Quinlan BD, Rangarajan ES, Pan A, Vanderheiden A, Suthar MS, Li W, Izard T, Rader C, Farzan M, Choe H. SARS-CoV-2 spike-protein D614G mutation increases virion spike density and infectivity. Nat Commun. 2020 Nov 26;11(1):6013. doi: 10.1038/s41467-020-19808-4. PMID: 33243994; PMCID: PMC7693302.

\section{Legend to figures}

451 Figure 1: Immunogenicity of VBI-2902a and VBI-2905a in mice. C57BL/6 mice, 8 per group, 452 received 2 IP injections 3 weeks apart, of VBI-2902a or VBI-2905a or a first injection of VBI-2902a 453 followed by a second injection of VBI-2905a (Heterologous boost), each containing $0.1 \mu \mathrm{g}$ of $\mathrm{S}$. 454 Blood was collected at day 14 after the second injection for monitoring of the humoral response. 
bioRxiv preprint doi: https://doi.org/10.1101/2021.09.28.462109; this version posted September 29, 2021. The copyright holder for this preprint (which was not certified by peer review) is the author/funder, who has granted bioRxiv a license to display the preprint in perpetuity. It is made available under aCC-BY-NC-ND 4.0 International license.

455 (a) Sera from each group were analyzed in PRNT assay with a 90\% threshold (PRNT90) using

Wu-1 virus and Beta virus as described in Material and Methods. GMT and results from two-tailed Mann-Whitney U-test are indicated. (b) Change in neutralization between Wu-1 and Beta viruses. Fold change was calculated for each serum as the ratio between reactivity to Ancestral and Beta RBD, Fold change in each group is indicated as the geometric mean preceeded by an arrow. Statistical analysis was determined using two tailed Wilcoxon test. (c) Ab binding titers were evaluated by ELISA using recombinant Delta RBD as described in Material and Methods. Statistical significance was determined by Kruskall-Wallis test.

Figure 2: Beta variant challenge in Syrian golden hamsters after immunization with VBI-

2902a and VBI-2905a. (a) Schematic representation of the challenge experiments. Four groups of 10 Syrian gold hamsters received 2 IM injections 3 weeks apart, of placebo saline buffer or VBI2902a or VBI-2905a or a first injection of VBI-2902a followed by a second injection of VBI-2905a, with $1 \mu \mathrm{g}$ of $\mathrm{S}$ per dose. Animals in Placebo groups received Saline buffer. Blood was collected 2 weeks after each injection. Three weeks after the last injection (day 42) hamsters were exposed to SARS-CoV-2 Beta virus at $1 \times 10^{5}$ TCID50 per animal via both nares. At 3 days post infection (dpi), 5 animals per groups were sacrificed for viral load analysis. The remaining animals were clinically evaluated daily until end of study at 14dpi. (b) Neutralization activity was measured by PRNT90 in

472 immunized groups; results are represented as PRNT90 EPT. GMT and statistical significance from 473 two tailed Friedman test are indicated (c) Hamsters were monitored daily for weight change. 474 Results are represented for each animal in each groups as kinetic of weight change from day 0 to 475 day 14 after infection. One animal from VBI-2905a group was sacrificed at day 7 because of worsening of clinical presentation after a fight in the cage. Significant days of weight loss relative to

477 Saline group $(p<0.005)$ are indicated. Statistical analysis was performed with unpaired non 478 parametric multiple t test using Holm-Šidák method.

479 Figure 3: Immunogenicity of trivalent VBI-2901a. Three groups of 10 mice were immunized with 4802 doses of $\mathrm{VBI}-2901 \mathrm{a}(01 \mathrm{a})$ or $\mathrm{VBI}-2902 \mathrm{a}(02 \mathrm{a})$ or VBI-2905a (05a) 3 weeks apart. Blood was 481 collected at day 14 after the last injection for monitoring of the humoral response. (a) Neutralization 
482 EPT measured by PRNT90 against Wu-1 virus and Beta variant. GMT are indicated above each

483 group. (b) Neutralization of pseudoparticles expressing S from Wu-1 virus, or Delta or Kappa 484 variants are represented as half-maximum inhibitory dilutions (Neutralization ID50). Geometric 485 means are indicated above each panel. Due to technical limitations, only 8 sera per groups were 486 tested against Wu-1 and Kappa pseudoparticles and 4 sera against Delta pseudoparticles. Sera 487 were randomly picked. (c) Ab binding titers measured in ELISA against recombinant RBD from Wu4881 ancestral virus, or Beta, Delta, and Kappa variants. 
a

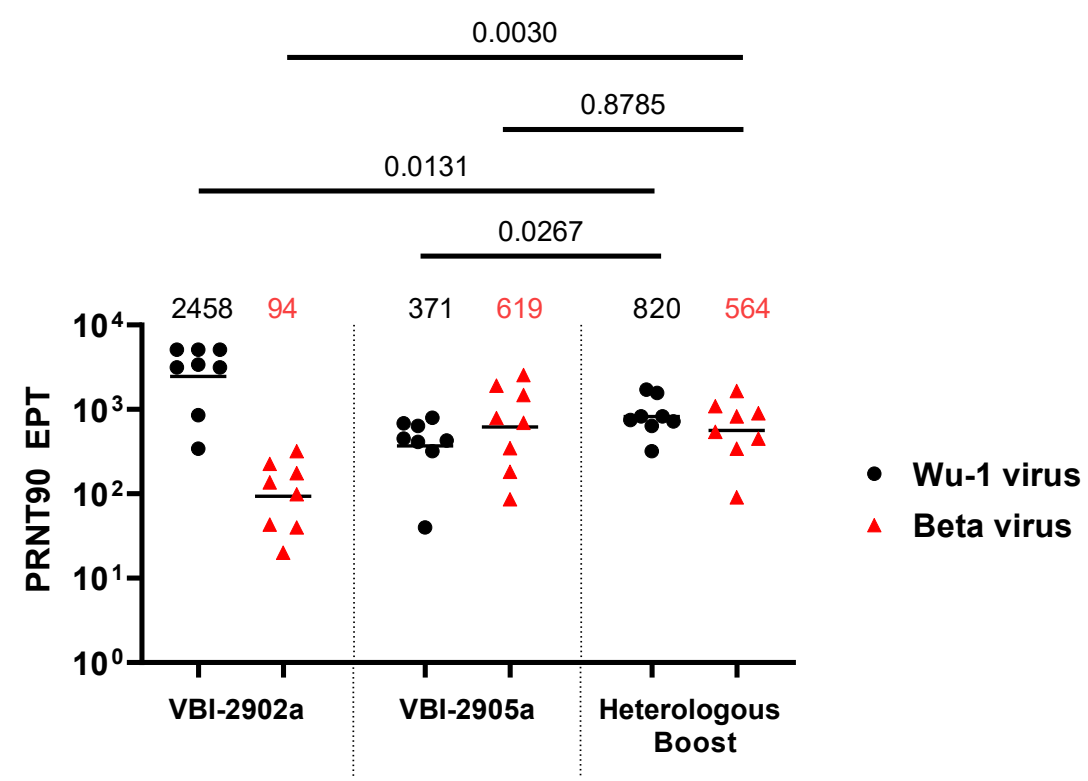

b

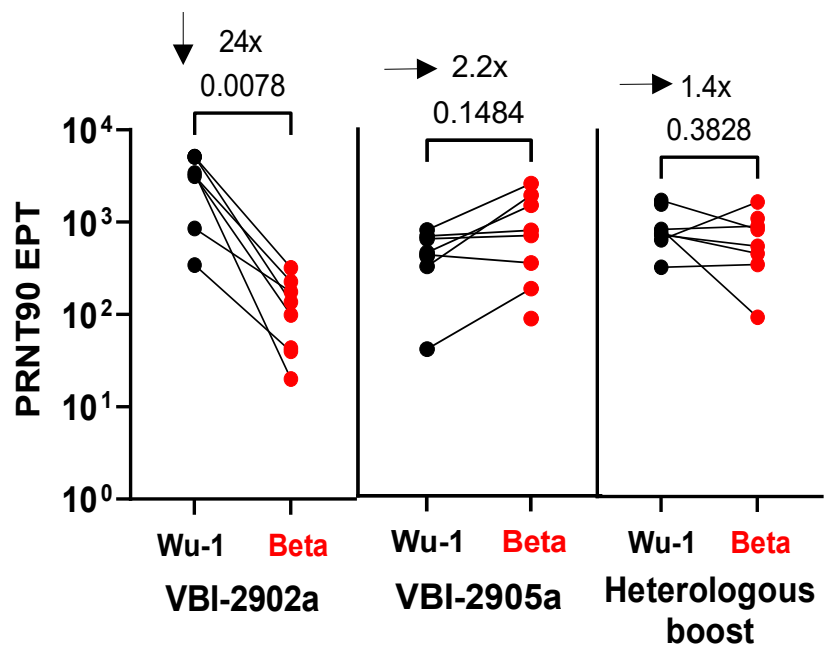

C

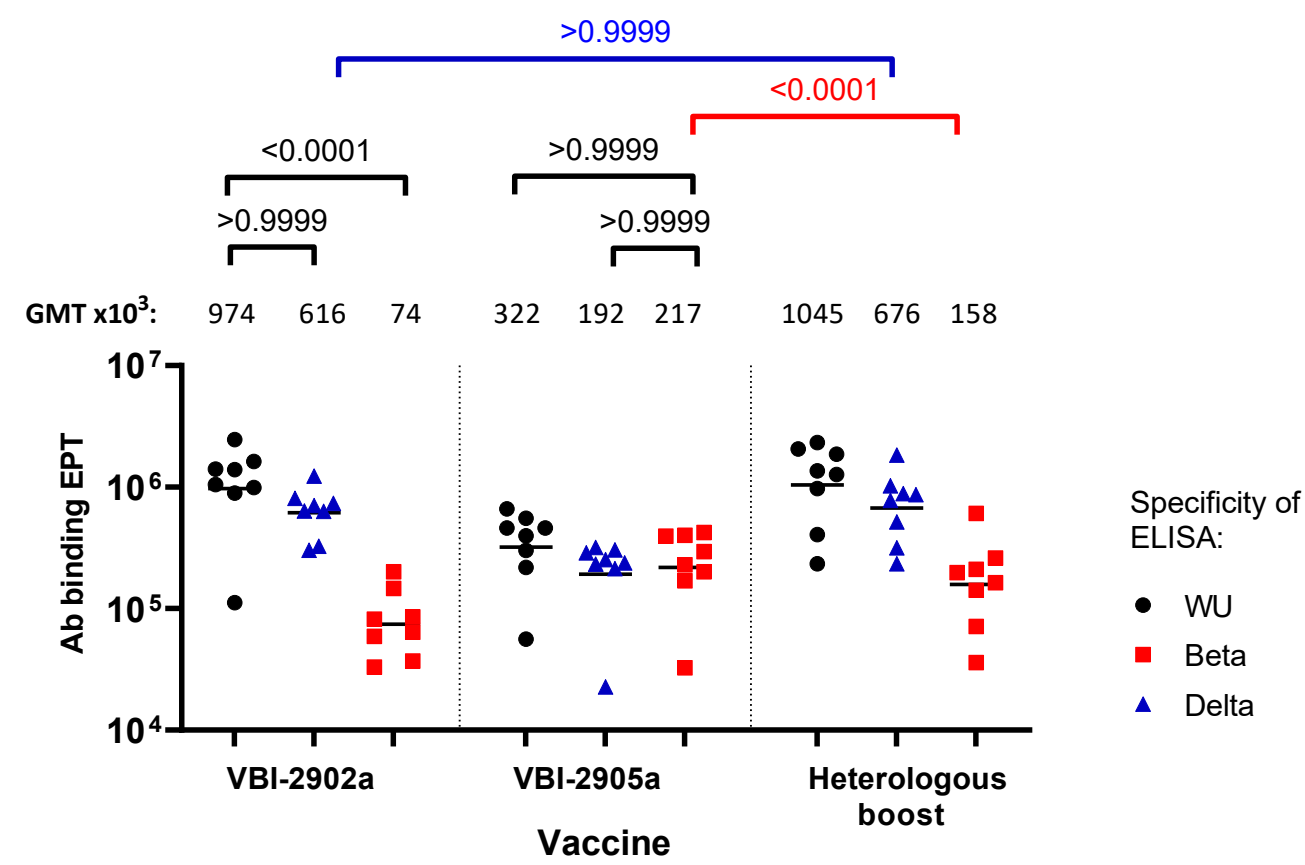




\section{FIGURE 2}

a

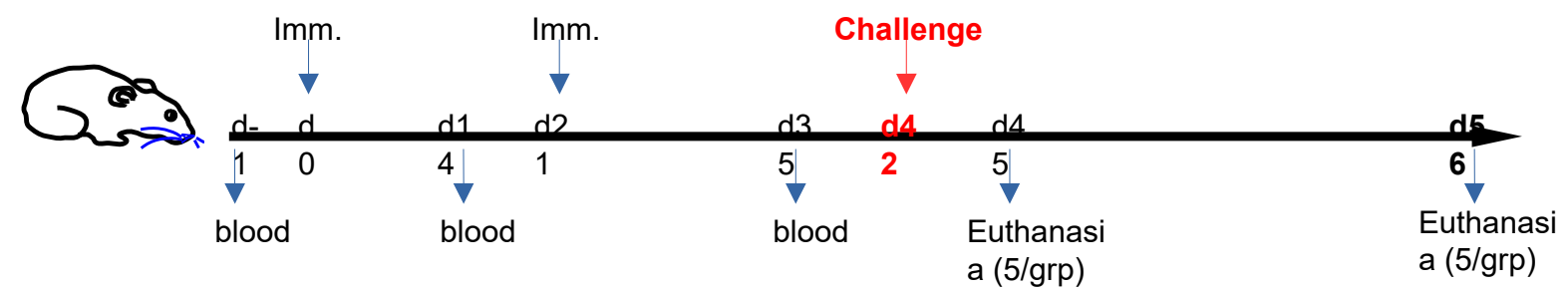

b

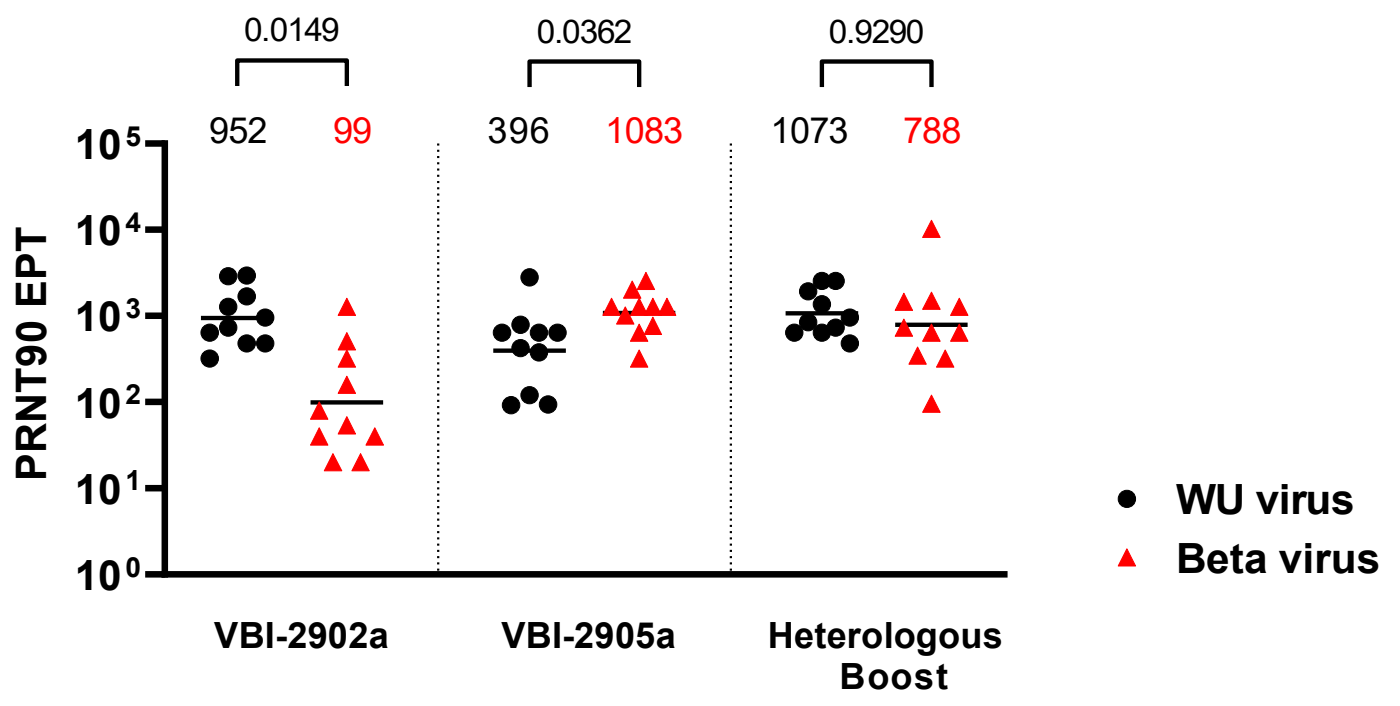

C

Saline

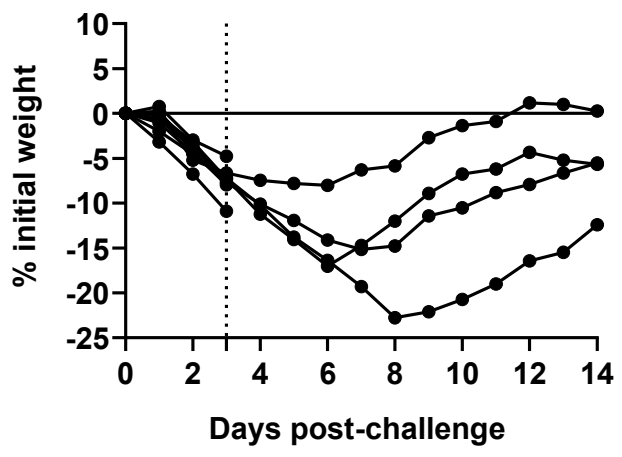

VBI-2902a

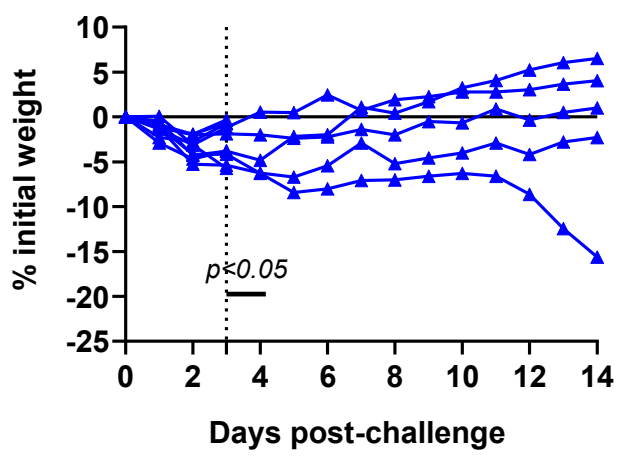

VBI-2905a

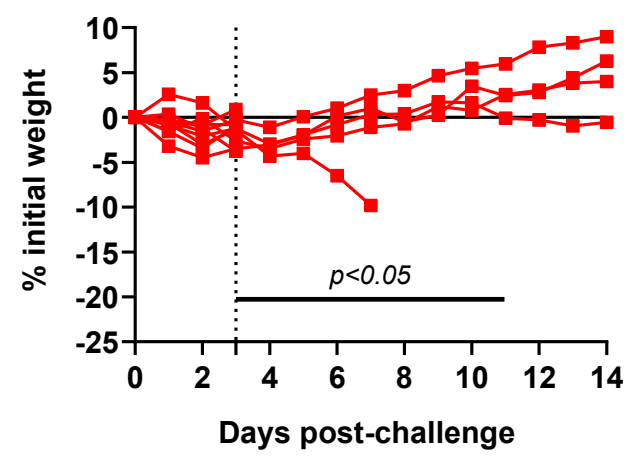

Heterologous boost

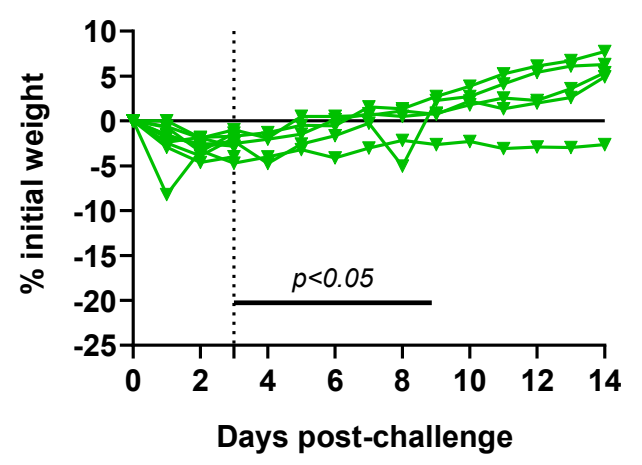


491 FIGURE 3

a

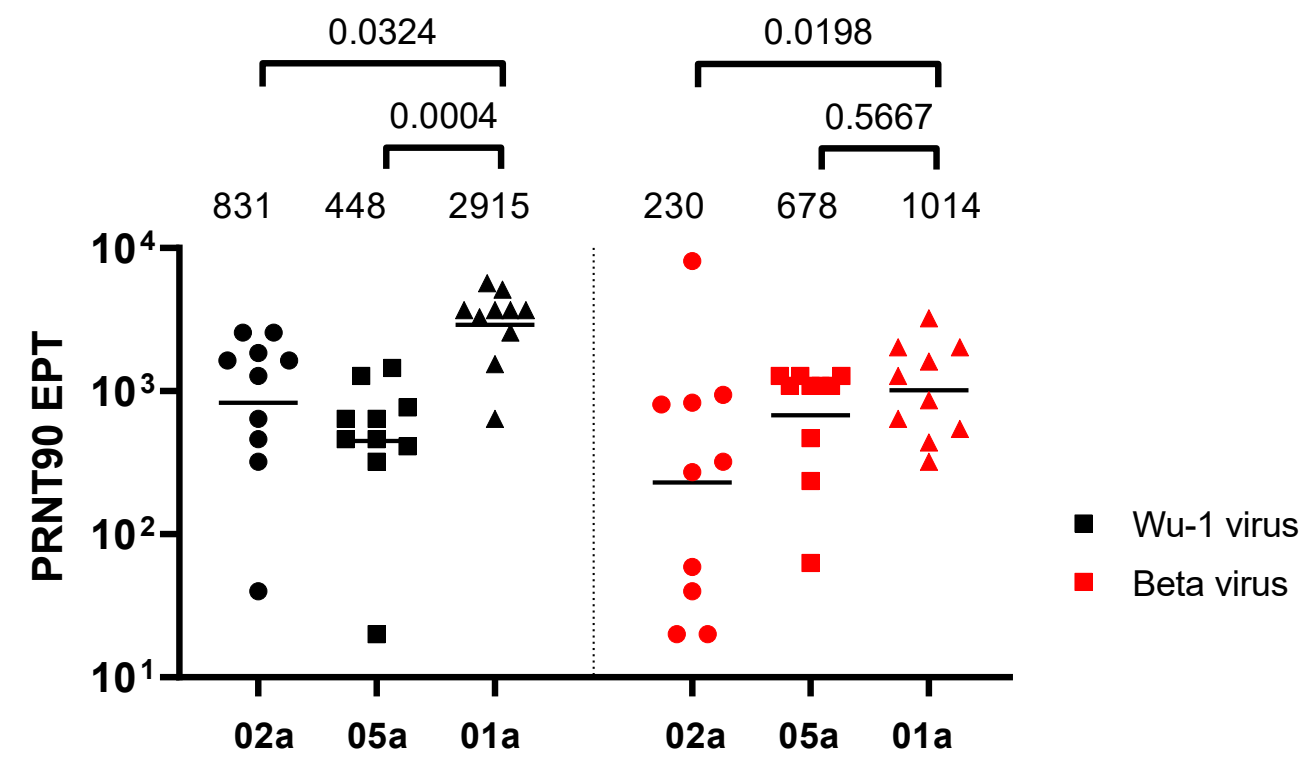

b

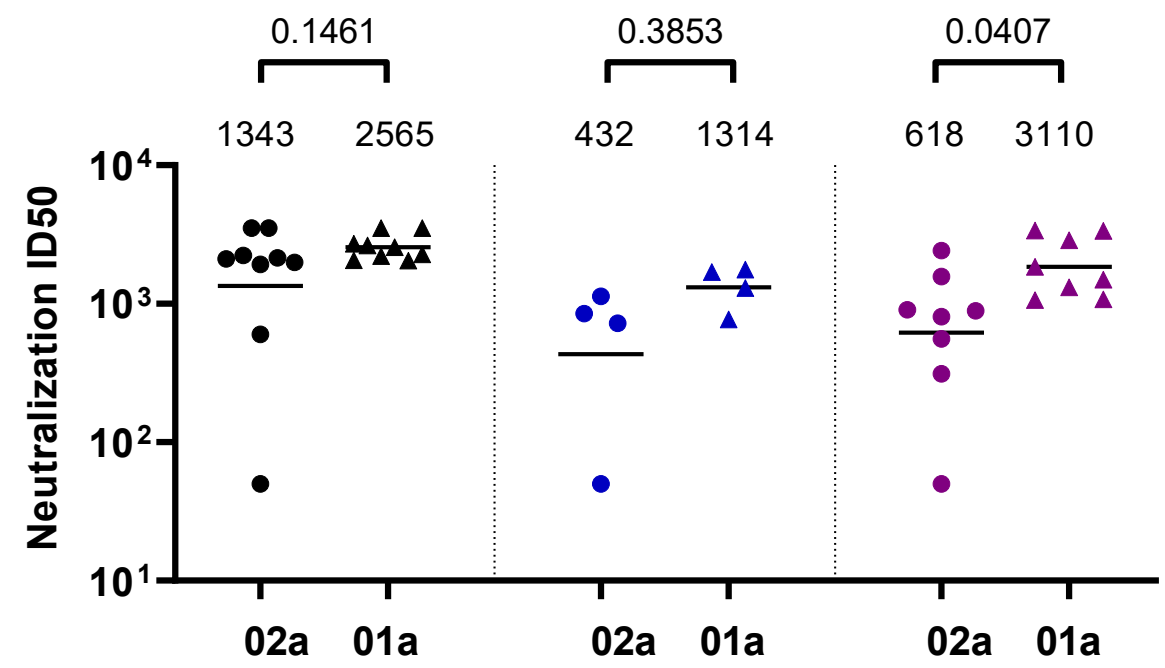

C

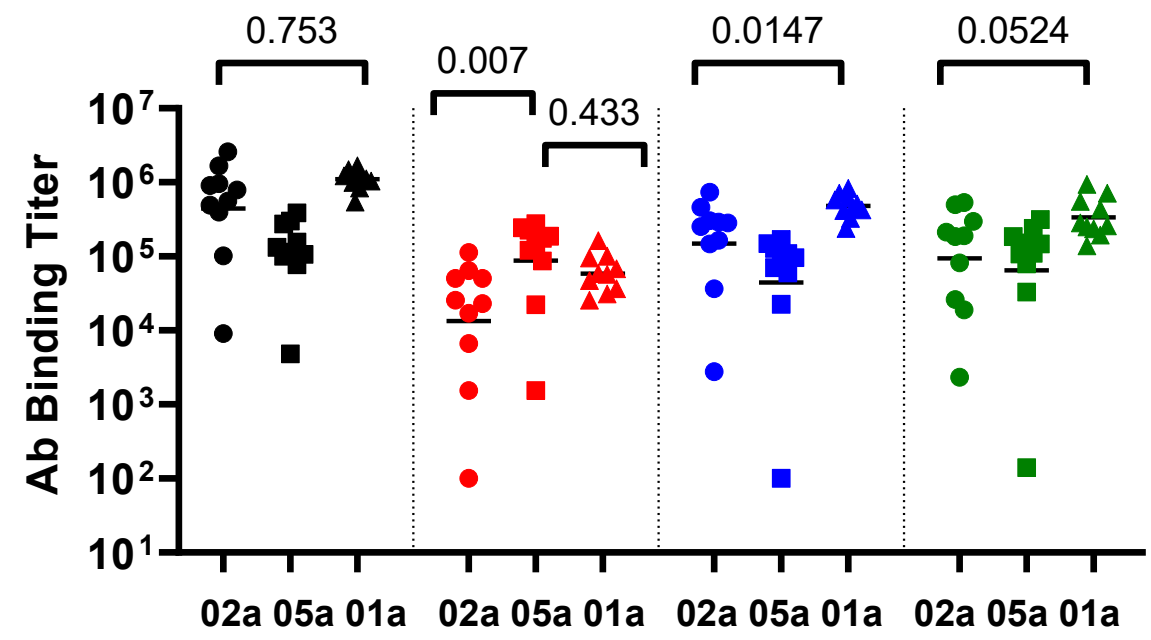

Specificity of ELISA

- Wu-1 RBD

- Beta RBD

- Delta RBD

- Lambda RBD

Pseudoparticles specificity

- Wu-1

- Delta

- Kappa 